\title{
GERMINACIÓN DE LAS SEMILLAS Y SUPERVIVENCIA DE LAS PLÁNTULAS DE ABIES PINSAPO BOISS.
}

\author{
Montserrat ARISTA
}

\begin{abstract}
RESUMEN. Germinación de las semillas y supervivencia de las plántulas de Abies pinsapo Boiss. Se estudió la germinación de las semillas y la supervivencia de las plántulas de Abies pinsapo Boiss. en tres zonas contrastadas dentro de la Reserva Natural de la Sierra de Grazalema (Cádiz): en un claro de bosque, en el sotobosque y fuera del pinsapar. La mejor germinación de las semillas se produjo fuera del bosque, pero las plántulas murieron rápidamente. Dentro del pinsapar, el claro fue la zona más idónea para la germinación y supervivencia de las plántulas.
\end{abstract}

Palabras clave. Abies pinsapo, germinación, supervivencia, Grazalema, Andalucía, España

\begin{abstract}
Seed germination and seedling survival of Abies pinsapo Boiss. Seed germination and seedling survival of Abies pinsapo Boiss. were studied in three diferent habitats of Sierra de Grazalema (Cádiz): in a gap-forest, under canopy and outside forest. Highest germination of seeds took place outside forest, but seedlings died quickly. Inside forest, the gap was the best place for germination of seeds and seedling survival.
\end{abstract}

Key words. Abies pinsapo, germination, survival, Grazalema, Andalucía, Spain.

\section{INTRODUCCIÓN}

El pinsapo (Abies pinsapo Boiss.), es uno de los endemismos vegetales más singulares de la Península Ibérica. Se distribuye por las Cordilleras Béticas entre los pisos bioclimáticos mesomediterráneo húmedo y supramediterráneo hiperhúmedo. Su área de distribución es relativamente pequeña $(1200 \mathrm{Ha}$. según Do Amaral Franco, 1986), lo que hace que sea considerado como una especie relicta. Hoy día, esta especie se encuentra protegida en Parques y Parajes Naturales para asegurar su conservación. En algunas de estas zonas se están llevando a cabo programas de reforestación para ampliar el área de distribución del pinsapo.

El estudio de la germinación de las semillas y supervivencia de las plántulas es una faceta de la biología de gran interés, ya que estos factores pueden condicionar la incorporación de individuos adultos a la población y con ello la regeneración de los bosques (Moloney, 1986). Estos aspectos que están ampliamente documentados en otros abetos (Barbour et al., 1990; Barton, 1930; Bates \& Roeser, 1928; Houle \& Payette, 1991; Jones et al., 1991), apenas han sido estudiados en Abies 
pinsapo (Arista et al., 1992). El objetivo del presente trabajo es determinar si el lugar donde se depositan las semillas en el bosque afecta de alguna forma a su germinación y posterior supervivencia, factores que, en la Sierra de Grazalema, podrían estar condicionando la distribución de Abies pinsapo.

\section{AREA DE ESTUDIO Y MÉTODOS}

El estudio se llevó a cabo en la Reserva de la Biosfera de la Sierra de Grazalema (Cádiz). En esta zona, el pinsapo forma un bosque espeso de unas $335 \mathrm{Ha}$, sobre la ladera $\mathrm{N}$ de la Sierra del Pinar, entre los 900 y 1400 msm. En las zonas más bajas de esta sierra, el pinsapo se mezcla con encinas (Quercus rotundifolia Lam.) en los suelos más secos y con quejigos (Quercus faginea Lam.) en los más húmedos y profundos, formando un bosque mixto. La superficie total que ocupa el pinsapo en esta ladera es de unas $700 \mathrm{Ha}$. Por todo el resto de la Reserva podemos encontrar indivíduos más o menos aislados junto con quejigos y algarrobos (Ceratonia siliqua L.) y rodeados por un espeso matorral.

En noviembre de 1991, momento en que se produce la dispersión de los piñones, se realizaron siembras en tres zonas de características muy diferentes, tanto dentro como fuera del pinsapar. Dentro de la masa de pinsapos se eligió un claro de bosque, donde la luz entra directamente durante buena parte del día y del año, y una zona cercana a la anterior pero debajo del dosel de ramas de pinsapos, en el sotobosque, donde no entra luz directa en ningún momento del día. Fuera del pinsapar, en el valle, se eligió una zona llana, donde además de llegar la luz durante todo el día existen otras condiciones diferentes a las que se dan dentro del bosque, tales como las edáficas, de temperatura o de humedad.

En cada una de las tres zonas descritas se sembraron 120 piñones separados en dos réplicas que se colocaron directamente sobre el suelo. Cada una de las réplicas se cubría con una caja confeccionada con malla plástica (20 x $20 \times 12 \mathrm{~cm}$ ) para evitar tanto la desaparición de los piñones como la llegada accidental de otros nuevos. A la vez que se sembraron estos piñones en el campo, en el laboratorio se pusieron otros a germinar en placas de Petri con papel de filtro estéril (10 por placa) y fueron regados periódicamente, sirviendo estas semillas como un control del porcentaje máximo de germinación.

Todos los piñones utilizados procedían de un sólo pinsapo del Puerto del Pinar y pesaron más de $60 \mathrm{mg}$, lo que garantiza que contienen embrión (Arista et al., 1992). Un piñón se consideró germinado cuando la longitud de la raíz fue mayor de $1 \mathrm{~mm}$. Las parcelas de estudio fueron visitadas periódicamente

Germinación (\%)

\begin{tabular}{lccc}
\hline \multicolumn{4}{c}{ Germinación $(\%)$} \\
\hline Zona & Réplica 1 & Réplica 2 & Réplica 1 + \\
Valle & 63.3 & 60 & 61.6 \\
Claro & 26.6 & 51.5 & 39.1 \\
Sotobosque & 0 & 0 & 0 \\
Laboratorio & 98.3 & 100 & 99.1 \\
\hline
\end{tabular}

Tabla 1: Porcentajes de germinación de los piñones de Abies pinsapo mayores de $60 \mathrm{mg}$ sembrados en diferentes zonas del pinsapar de Grazalema y en el laboratorio. Todos los piñones proceden del mismo árbol. Se muestran los resultados obtenidos para cada una de las dos réplicas y para el total de la muestra. El número de piñones por réplica es 60 . 
hasta seis meses después del comienzo del experimento, anotando en cada ocasión el número de piñones que había germinado y la supervivencia de las plántulas.

\section{RESULTADOS}

El porcentaje de germinación de los piñones dependió en gran medida del lugar donde se colocó la muestra (tab. 1). El mayor porcentaje se produjo fuera del pinsapar, en el valle con un $61.6 \%$, seguido por el claro con un $39 \%$, siendo estos porcentajes significativamente distintos $\left(\chi^{2}=6, p<0.05\right)$. En el sotobosque del pinsapar no germinó ninguno de los 120 piñones sembrados. En el control del laboratorio el porcentaje de germinación fue muy alto, prácticamente del $100 \%$.

Se apreciaron también diferencias entre zonas en cuanto a la velocidad de la germinación; así, en el laboratorio los piñones comenzaron a germinar 42 días después de la siembra, mientras que en el campo (tanto en el valle como en el claro) ésto no ocurrió hasta tres meses después de haber sido sembrados.
Los porcentajes de supervivencia de las plántulas desde su emergencia hasta transcurridos seis meses se muestran en la tabla 2 . Como puede observarse, en cualquier momento el mayor porcentaje de supervivencia se dio en el claro del bosque. Las plántulas del valle murieron con gran rapidez, y sólo en los 15 primeros días la mortalidad llego al 78\%. En el mismo periodo de tiempo, la mortalidad en el claro fue significativamente inferior con sólo el $43 \%\left(\chi^{2}=15.6, p<0.05\right)$. Transcurridos seis meses en el valle no sobrevivía ninguna plántula, mientras que en el claro aún quedaban vivas cuatro.

\section{DISCUSIÓN}

Aunque la dormancia a largo plazo que permitiría la formación de un banco de semillas, quedó descartada en Abies pinsapo (Arista et al., 1992), los 42 días que tardaron en germinar los piñones en el laboratorio, sugiere que éstos necesitan un breve período de descanso tras ser dispersados. Período que podría ser debido a una incompleta maduración de las semillas, tal y como ocurre en otras especies

\begin{tabular}{lccccc}
\hline & & \multicolumn{3}{c}{ Supervivencia (\%) } \\
\hline \multirow{2}{*}{ Zona } & Réplica & $\mathrm{n}$ & 15 días & 30 días & 180 días \\
& & & & & \\
Valle & 1 & 38 & 23.6 & 23.6 & 0 \\
& 2 & 36 & 19.4 & 13.8 & 0 \\
Claro bosque & $1+2$ & 74 & 21.6 & 18.9 & 0 \\
& 1 & 16 & 37.5 & 37.5 & 6.2 \\
& $1+2$ & 31 & 67.7 & 64.5 & 9.6 \\
Laboratorio & 1 & 59 & 57.4 & 55.3 & 8.5 \\
& 2 & 60 & 100 & 100 & 100 \\
& $1+2$ & 119 & 100 & 100 & 100 \\
& & & & 100 & 100 \\
\hline
\end{tabular}

Tabla 2: Porcentajes de supervivencia de plántulas procedentes de piñones, mayores de $60 \mathrm{mg}$, germinados en distintas zonas de la Reserva de Grazalema, a los 15, 30 y 180 días tras la germinación. El primer censo corresponde a finales de marzo, el segundo a mediados de abril y el tercero a finales de septiembre. Se muestran los resultados obtenidos para cada una de las dos réplicas y para el total de la muestra. $\mathrm{n}$ es el número de plántulas inicial. 
(Harper, 1977). En el campo, los piñones tardaron el doble de tiempo en germinar, posiblemente como consecuencia de factores ambientales. El retraso o adelanto de la germinación dependiendo de condiciones ambientales como las temperaturas, ha sido anteriormente descrito en otras coníferas como Abies lasiocarpa, Picea engelmanii (Knapp \& Smith, 1982) y Araucaria angustifolia (Ferreira \& Handro, 1979).

Las diferentes tasas de germinación de los piñones obtenidas en las tres zonas, pudieron ser debidas principalmente a la presencia o ausencia de humedad en el suelo más que a las diferencias de luz entre ellas. En otras especies forestales, las semillas son muy sensibles a la composición lumínica (fotoblastismo) y sólo germinan en determinadas zonas del bosque (Vazquez-Yanes y Orozco-Segovia, 1990; Vazquez-Yanes et al., 1990). Pero, aunque la luminosidad de la zona pueda influir en la mayor o menor germinación de las semillas de A. pinsapo, nos consta que éstas son capaces de germinar en el sotobosque con condiciones lumínicas muy bajas, ya que en esta zona aparecen plántulas (Arista, 1993). Otra explicación alternativa podría ser una inhibición de la germinación provocada por alelopatía tal y como ocurre en muchas otras especies (Taylor \& Shaw, 1983; Wardle et al., 1991), sin embargo estudios anteriores mostraron que la tierra procedente del sotobosque del pinsapar tiene un efecto débil sobre la tasa de germinación de las semillas de pinsapo (Arista et al., 1992). La diferencia más notable entre zonas además de la luz, fue la humedad del suelo, y posiblemente ésta haya sido la causa de que en el sotobosque no germinase ningún piñón.

Fuera de la masa de pinsapos, las semillas germinan bien o incluso mejor que dentro del pinsapar (tab. 1). Sin embargo las plántulas murieron rápidamente en cuanto comenzó la primavera y el verano, seguramente como consecuencia del estrés hídrico ya que estaban a pleno sol. En otros abetos como Abies concolor y $A$. magnifica, la sequía es también una fuente importante de mortalidad de las plántulas durante su primer año de vida (Barbour et al., 1990). El escaso número de plántulas que sobreviven cuando aparecen las altas temperaturas primaverales y veraniegas fuera del pinsapar, podría ser una de las causas del escaso número de individuos juveniles que se encuentran en estas zonas. De esta manera, a pesar de que las semillas de pinsapo germinan bien fuera del pinsapar, la alta mortalidad de las plántulas podría limitar la incorporación de individuos adultos a la población, siendo una de las causas responsables de la distribución de Abies pinsapo en la Sierra de Grazalema.

Estos hechos pueden tener gran importancia desde el punto de vista del manejo de la especie, ya que la probabilidad combinada de que una semilla viable germine y la plántula originada sobreviva hasta alcanzar los seis meses de edad es de 0.03 en el claro de bosque, o dicho de otra manera, un $3 \%$ de las semillas que caen en un claro dan lugar a una plántula de seis meses, mientras que fuera del pinsapar la probabilidad es prácticamente de cero. En el caso de que se realicen repoblaciones, las plántulas que sean sembradas en lugares muy soleados fuera del bosque posiblemente no consigan sobrevivir, siendo más efectivas las siembras en zonas húmedas.

AGRADECIMIENTOS Agradezco a los Drs. S. Talavera, J. Herrera y P. L. Ortiz los comentarios y aportaciones al manuscrito original. La Agencia de Medio Ambiente (Junta de Andalucía) permitió realizar el trabajo en la zona de Reserva (Parque Natural Sierra de Grazalema).

\section{BIBLIOGRAFÍA}

ARISTA, M. -1993- Biología del pinsapo (Abies pinsapo Boiss.). Tesis Doctoral. Universidad de Sevilla. 
ARISTA, M., S. TALAVERA y J. HERRERA 1992- Viabilidad y germinación de las semillas de Abies pinsapo Boiss. Acta. Bot. Malacitana, 17: 223-228.

BARBOUR, M.G., B.M. PAVLIK \& J.A. ANTOS -1990- Seedling growth and survival of red and white fir in a Sierra Nevada ecotone. Amer. $J$. Bot., 77: 927-938.

BARTON, L.V. -1930- Hastening the germination of some coniferous seeds. Amer. J. Bot., 17: 88115.

BATES, C.G. \& J. Jr. ROESER -1928- Light intensities required for growth of coniferous seedlings. Amer. J. Bot., 15: 185-194.

DO AMARAL FRANCO -1986-Pinaceae en CASTROVIEJO \& al. (eds.) Flora Ibérica Vol. I. C.S.I.C. Madrid.

FERREIRA, A.G. \& W. HANDRO-1979- Aspects of seed germination in Araucaria angustifolia (Bert.) O. Ktre. Revta. Brasil. Bot., 2(1): 7 -14.

HARPER, J.L. -1977- Population biology of plants. Academic Press. London.

HOULE, G. \& S. PAYETTE-1991-Seed dynamics of Abies balsamea and Acer saccharum in a decidous forest of Northeastern North America. Amer. J. Bot., 78(7): 895-905.

JONES, S.K., Y.K. SAMUEL \& P.G. GOSLING 1991- The effect of soaking and prechilling on the germination of noble fir seeds. Seed Sci. \& Technol, 19: 287-293.

KNAPP, A.K. \& W.K. SMITH -1982- Factors influencing understory seedling establishment of engelmann spruce (Picea engelmanii) and subalpine fir (Abies lasiocarpa) in southeastern Wyoming. Can. J. Bot., 60: 2753-2761.

MOLONEY, K.A. -1986- Wave and nonwave regeneration processes in a subalpine forest. Can. J. Bot., 64: 341-349.

TAYLOR, R.J. \& D.C. SHAW -1983- Allelopathic effects of engelmann spruce bark stilbenes and tannin-stilbene combinations on seed ger mination and seedling growth of selected conifers. Can. J. Bot., 61: 279-289.
VAZQUEZ-YANES, C.\& A. OROZCO SEGOVIA -1990- Ecological significance of light controlled seed germination in two contrasting tropical habitats. Oecologia, 83: 171-175.

VAZQUEZ-YANES, C., A. OROZCO-SEGOVIA, E. RINCON, M.E. SANCHEZ-CORONADO, P. HUANTE, J.R. TOLEDO \& V.L. BARRADAS -1990- Light beneath the litter in a tropical forest: effect on seed germination. Ecology, 71(5): 1952-1958.

WARDLE, D.A., M. AHMED \& K.S. NICHOLSON -1991- Allelopathic influence of nodding thistle (Carduus nutans L.) seeds on germination and radicle growth of pasture plants. New Zealand J. Ag. Res., 34: 185-191.

Aceptado para su publicación en Julio de 1993

Dirección de la autora. Departamento de Biología Vegetal y Ecología. Universidad de Sevilla. Apartado 1095. 41080 Sevilla. 